\title{
Der Sanktionsbedarf von Facebook-Inhalten aus Sicht von Nutzerlnnen und seine Determinanten
}

Teresa K. Naab

In den sozialen Medien können NutzerInnen, die keine institutionalisierte Ausbildung über die Bedeutung der Meinungsfreibeit und ibre Grenzen erbalten haben, von anderen NutzerInnen produzierte Medieninhalte sanktionieren. Diese Sanktionen ergänzen die Regulierung durch Plattformanbieter und die rechtsstaatliche Regulierung. Damit eröffnet sich ein neues Forschungsfeld über die Potenziale und Herausforderungen des Sanktionsbedarfs von Medieninhalten aus Sicht von NutzerInnen. In einer standardisierten Befragung von 265 Facebook-Usern wird untersucht, welche Merkmale und Einstellungen beeinflussen, ob NutzerInnen einen Facebook-Inhalt negativ sanktionieren würden oder Sanktionen vom Plattformbetreiber oder vom Staat erwarten. Es zeigen sich teilweise Parallelen zur den Forschungsergebnissen, die in Studien zu traditionellen Medien gewonnen wurden: Die Einschätzung der Medienbotschaft als gefährlich, geringere Facebook-Nutzung und ein niedrigeres Bildungsniveau geben mit einer hoben Einschätzung des Sanktionsbedarfs aus Sicht der NutzerInnen einher. Andere Faktoren (Alter, Geschlecht, politische Orientierung, Religiosität und vermutete Wirkung des Facebook-Inhalts) baben dagegen keine oder nur indirekte Effekte.

Schlagwörter: Regulierung, Sanktionen, Social Media, Facebook, Befragung

Durch die Verbreitung des Internets und insbesondere der sozialen Medien erwachsen umfangreiche öffentliche Äußerungsmöglichkeiten für Laien. Damit gehen jedoch auch vermehrt problematische Botschaften einher und die Meinungsfreiheit kollidiert immer wieder mit anderen Grundrechten. In den traditionellen Massenmedien (in Deutschland) übernimmt die Kontrolle regulierungsbedürftiger Äußerungen a prioi das publizierende Medium und post hoc die rechtsstaatliche Autorität. Die Verbreitung neuer Medien erfordert jedoch neue Formen der Beobachtung problematischer Inhalte (Naab, 2012): Durch die stetigen technischen Entwicklungen kann eine aktuelle Gesetzeslage, die auch neue Anwendungen, Nutzungsformen und Geschäftsmodelle berücksichtigt, kaum gewährleistet werden. Internetinhalte werden Rechtsgrenzen überschreitend verbreitet und machen eine international abgestimmte Regulierung notwendig. Die KommunikatorInnen, Plattformen und Inhalte haben sich vervielfacht und dezentralisiert. Dies bringt auch die Medienunternehmen an ihre Leistungsgrenze bei der Kontrolle der Inhalte.

In rückkanalfähigen Medien treten die NutzerInnen als weitere Akteursgruppe mit Einfluss auf die Sanktionierung unangemessener Inhalte hinzu. Sie können mit verschiedenen Sanktionsmitteln gegen die Äußerungen anderer vorgehen und sie an eine Autorität melden. Allerdings sind wissenschaftliche und insbesondere empirische Betrachtung dieses Phänomens selten. Zwar liegen Studien vor zu den abstrakten Einschätzungen von Laien, ob ein Medieninhalt verboten werden sollte (z. B. Lee \& Tamborini, 2005; Salwen \& Dupagne, 1999; Youn, Faber \& Shah, 2000). Allerdings gibt es nur wenige Untersuchungen von Personengruppen, die selbst in der Position sind, Maßnahmen gegen vermeintlich unangemessene Kommunikate zu ergreifen (zu PolitikerInnen: Dohle \& Bernhard, 2013; Sullivan, Walsh, Shamir, Barnum \& Gibson, 1993; zu AktivistInnen: 
Bahry, Boaz \& Gordon, 1997; Gibson \& Bingham, 1983; zu Eltern: Hoffner \& Buchanan, 2002).

Das vorliegende Manuskript betrachtet deshalb die Einschätzungen von NutzerInnen, ob ein Social Media-Inhalt sanktionsbedürftig ist. Es stellt Sanktionsmittel der NutzerInnen, der Plattformanbieter und des Staates dar. So wird erstmals umrissen, welche Mittel NutzerInnen als adäquat zur Regulierung von Inhalten Anderer ansehen. Die nutzerseitige Einschätzung bildet in einer demokratischen Gesellschaft eine Grundlage für die Legitimation und Akzeptanz rechtlicher Regulierung. Denn welche Äußerungen schützenswert sind und wie auf problematische Botschaften reagiert werden sollte, bedarf einer ständigen Aushandlung. Anschließend arbeitet das Manuskript auf Basis von Literatur zu traditionellen Medien potenzielle Einflussfaktoren auf die Angemessenheit von Sanktionen aus Nutzersicht heraus und prüft in einer Studie mit Facebook-Inhalten, welche Determinanten den von NutzerInnen empfundenen Sanktionsbedarf erklären können. Dies gibt zum einen erste empirische Hinweise, warum MediennutzerInnen sich mehr oder weniger tolerant gegenüber den von anderen produzierten Botschaften verhalten. Zum anderen liefert es Erkenntnisse über die Übertragbarkeit von Wissen aus traditionellen Medienkontexten auf soziale Medien. Damit trägt es zum wissenschaftlichen Verständnis der Wahrnehmung und Beurteilung von Inhalten verschiedener Mediengattungen bei.

\section{Sanktionsmittel von NutzerInnen, Plattformanbietern und Staat}

Anders als die Rezipierenden klassischer Massenmedien ohne nennenswerte Rückkanäle sind die NutzerInnen sozialer Medien in der Lage, ihre Wertschätzung bestimmter Inhalte zurückzuspielen. Sie können Medienbotschaften, die von den Normen ihrer sozialen Gruppe abweichen, negativ sanktionieren (ausführlich Gibbs, 1981). Je nach Plattform stehen ihnen verschiedene Reaktionsmöglichkeiten offen. Sie können mit Bewertungs-Buttons, öffentlichen Antworten oder nicht-öffentlichen Nachrichten an die KommunikatorInnen ihr Missfallen ausdrücken und damit ihre Einschätzung über die Zulässigkeit eines Kommunikationsinhalts abgeben (Naab, in Druck; auch Döring, 2001). So bestrafen NutzerInnen andere User für deviante Botschaften. Diese sozialen Sanktionen können alle Nutzenden ausüben, ohne eine besondere Machtstellung auf der Medienplattform innezuhaben. Allerdings hängt ihre Wirksamkeit von der Reputation der Sanktionierenden und der Unterstützung durch andere Nutzende ab.

Sofern Plattformbetreiber in gewissem Maße die Kontrolle aus der Hand geben, profitieren sie von der Mitarbeit der NutzerInnen bei der Beurteilung der großen Anzahl von Beiträgen. Auch die Gefahr von Konflikten durch ein Machtungleichgewicht zwischen Nutzenden und professionellen ModeratorInnen wird geringer. NutzerInnen in den Prozess der Regulierung von Medienbotschaften einzubinden, bestärkt außerdem Hoffnungen, dass die Inhaltskontrolle von verschiedenen Akteuren ausgeht und so das Potenzial für die Unterdrückung bestimmter Meinungen reduziert wird. Allerdings ist nicht sichergestellt, dass NutzerInnen entsprechend demokratischer Prinzipien sanktionieren. Eine gleichberechtigte Repräsentation verschiedener Standpunkte ist deshalb nicht notwendigerweise gewährleistet, zumal nutzerseitige Sanktionen auch eingesetzt werden können, um gezielt unliebsame Meinungen zu verdrängen (Crawford \& Gillespie, 2014; Naab, in Druck).

Im Gegensatz zu den NutzerInnen verfügen die Betreiber von Social Media-Plattformen über technische Sanktionsmöglichkeiten (Bruckman, Danis, Lampe, Sternberg \& Waldron, 2006; Döring, 2001). Die Plattformanbieter können unangemessene Äußerungen löschen, verändern und User sperren (z. B. Goodman, 2013; Gurzick, White, 
Lutters \& Boot, 2009). Diese Sanktionen basieren auf einem Machtungleichgewicht zwischen den Kontrollierenden und den Devianten, die meist keine Gegenmaßnahmen ergreifen können. Betreiber stehen deshalb immer wieder in der Kritik wegen mangelnder Transparenz bei der Beschränkung von Inhalten (z. B. Weck, 2012).

Ein Eingreifen des Staates ist ein demokratisch legitimierter Regulierungsschritt. Eine Zensur im Sinne einer Vorabprüfung durch staatliche Instanzen findet in Deutschland nicht statt. Nicht ausgeschlossen durch das Zensurverbot in Artikel 5 des Grundgesetzes ist jedoch die nachträgliche Prüfung und ggf. das Verbot einzelner Äußerungen. Über die Maßnahmen gegen die weitere Verbreitung rechtswidriger Inhalte hinaus obliegt der rechtsstaatlichen Autorität die strafrechtliche Verfolgung der Verantwortlichen (Buchloh, 2003).

Soziale, technische und rechtsstaatliche Kontrollmechanismen der drei Akteursgruppen verstärken und ergänzen sich in einer demokratischen Gesellschaft gegenseitig: Bei einer effektiven sozialen Kontrolle ist die Notwendigkeit rechtsstaatlicher Zwangsregulierung reduziert. Gleichzeitig können die Mitglieder einer Gesellschaft auf institutionalisierte und demokratisch legitimierte Sanktionen vertrauen, wenn ihre sozialen und technischen Sanktionen nicht greifen. Aufgrund der eingeschränkten Wirksamkeit sozialer Kontrolle durch andere NutzerInnen finden sich auf vielen Angeboten zusätzlich Melde-Buttons, mit denen die User ModeratorInnen über vermeintliche Verstöße informieren können. Davon profitieren auch die Betreiber, denn die Vielzahl der KommunikatorInnen und Botschaften auf manchen Plattformen stellt sie vor große Herausforderungen bei der zeitnahen und effektiven Kontrolle. Sie binden deshalb NutzerInnen ein, die so wiederum größere indirekte Sanktionsmöglichkeiten erhalten. Ob der eingangs genannten Entwicklungen in den neuen im Vergleich zu den traditionellen Medien ist die rechtliche Regulierung eingeschränkt. Für die Strafverfolgungsbehörden ist deshalb eine enge Zusammenarbeit mit den Plattformbetreibern unumgänglich. Auch die NutzerInnen können rechtliche Sanktionen anregen, indem sie sich direkt oder über Mittler an die Strafverfolgungsbehörden wenden (zum Vorgehen Jugendschutz.net, 2014).

Das vorliegende Manuskript konzentriert sich auf die Einschätzung des Sanktionsbedarfs von Inhalten in sozialen Medien durch die NutzerInnen. Ob ihrer Komplementarität betrachtet es die Angemessenheit von Sanktionsmitteln aller drei Akteursgruppen aus Nutzersicht.

Empirische Literatur konzentriert sich vorrangig auf die Zustimmung von Laien zu Maßnahmen der Regierung, beispielsweise ob bestimmte Inhalte von der Regierung verboten, ihre KommunikatorInnen bestraft (Lambe, 2002; Neuwirth \& Frederick, 2002; Salwen \& Dupagne, 1999) oder Inhalte vorabgeprüft werden sollten (Huh, Delorme \& Reid, 2006; Thompson, Chaffee \& Oshagan, 1990). Teilweise wird nach dem Engagement der NutzerInnen gefragt, die Regierung auf Handlungsbedarf hinzuweisen (Zhao \& Cai, 2008). Einige Studien erfragen die Zustimmung der NutzerInnen zu Sanktionen durch Medienunternehmen oder Internetprovider (McLeod, Detenber \& Eveland, 2001; McLeod, Eveland \& Nathanson, 1997; Wei \& Lo, 2007) oder eigene Handlungsaufforderungen an diese (Lo \& Wei, 2002; Rojas, Shah \& Faber, 1996). In wenigen Fällen werden die Zustimmung zu Handlungen anderer Laien (Wei \& Lo, 2007) oder hypothetische eigene Sanktionsmaßnahmen der NutzerInnen erfragt, wie etwa an einer Demonstration gegen Internetpornografie teilzunehmen (Lo \& Wei, 2002; Rojas et al., 1996). Integrierende Studien, die den wahrgenommenen Sanktionsbedarf von Medieninhalten durch die verschiedenen, komplementären Sanktionsmöglichkeiten erfassen und die Feedbackmöglichkeiten sozialer Medien aufgreifen, sind nicht bekannt. 


\section{Einflüsse auf den empfundenen Sanktionsbedarf}

\subsection{Forschungsstand}

Berührt eine Meinungsäußerung eine grundsätzliche Werthaltung von Rezipierenden, wird ihre (Nicht-)Toleranz als nicht situationsabhängig verhandelbar wahrgenommen (Gunther, 1995; Huh et al., 2006; Lee \& Tamborini, 2005; Lo \& Wei, 2002; Price, Tewksbury \& Huang, 1998). Menschen tendieren umso stärker zu Einschränkungen der Äußerungsfreiheit, je mehr sie sich von KommunikatorInnen bedroht fühlen (Bahry et al., 1997; Gibson, 2006; Hurwitz \& Mondak, 2002; Stupi, Chiricos \& Gertz, 2014). Die eingeschätzte Bedrohung umfasst je nach untersuchter Medienbotschaft Aspekte wie die vermeintliche Gefäbrlichkeit, Schädlichkeit, Unerwünschtheit oder wie unsozial oder unmoralisch das Dargestellte ist (Lambe \& McLeod, 2005; McLeod et al., 2001; Paek, Lambe \& McLeod, 2008; Salwen \& Dupagne, 1999).

Mehrere AutorInnen zeigen, dass die vermutete Wirkung von Botschaften auf andere und auf die eigene Person die Sanktionsbereitschaft gegenüber als negativ eingeschätzten Inhalten beeinflusst (Bernhard \& Dohle, 2014; Dohle \& Bernhard, 2013; Gunther, 1995; Lee \& Tamborini, 2005; Lo \& Wei, 2002; McLeod et al., 2001; Thompson et al., 1990; Wei \& Lo, 2007; Wei, Lo \& Lu, 2010; Youn et al., 2000). ${ }^{1}$

Während die eingeschätzte Gefährlichkeit einer Botschaft die grundsätzliche Einschätzung eines Kommunikationsinhalts oder Kommunikators darstellt, bezieht sich die vermutete Wirkung auf das Potenzial, dass die konkret über ein Medium transportierte Botschaft beim Publikum entfalten kann. Mit einer wahrgenommenen Gefahr geht nicht notwendigerweise eine hohe Wirkvermutung einher, weil letztere von weiteren Aspekten beeinflusst werden kann und Befragte vermeintlich besondere Resistenz gegen problematische Botschaften entwickeln (Lambe \& McLeod, 2005; McLeod et al., 2001; Paek et al., 2008; Salwen \& Dupagne, 1999).

Personen, die Botschaften bestimmter Thematik in den Medien nutzen, zeigen eine geringere Bereitschaft zu negativen Sanktionen gegenüber diesen Botschaften als NichtNutzerInnen (Gunther, 1995; Lee \& Tamborini, 2005; McLeod et al., 2001; Paek et al., 2008; für abweichende Ergebnisse Wei \& Lo, 2007; Wu \& Koo, 2001). Einen Effekt der allgemeinen Nutzung eines Mediums unabhängig von den zur Debatte stehenden Inhalten finden Trepte, Reinecke und Behr (2008). Sie zeigen, dass aktive User von Web 2.0-Anwendungen sich eher für Restriktionen in Weblogs stark machen.

Einflüsse verschiedener Persönlichkeitsmerkmale auf die Einstellung zur Meinungsfreiheit und gegenüber konkreten Medienbotschaften sind empirisch belegt. Für traditionelle Medien stützt die Forschungsliteratur die Annahme, dass eine Zustimmung zur Meinungsfreibeit im Allgemeinen zu einer geringen Sanktionsneigung führt. Menschen, die Meinungsfreiheit höher schätzen, sind eher bereit, sie auch im Falle ungeliebter oder extremer Äußerungen und KommunikatorInnen zuzugestehen (Davis, 1990; Gunther, 1995; Lambe, 2004; Marcus, Sullivan, Theiss-Morse \& Wood, 1995; Paek et al., 2008; Price et al., 1998; Sullivan, Piereson \& Marcus, 1982; Zhao \& Cai, 2008).

Suedfeld, Steel und Schmidt (1994) zeigen, dass Konservative eher einen Standpunkt für die Regulierung verschiedener Inhalte einnehmen (Dohle \& Bernhard, 2013; McLeod

1 Ein häufig untersuchter Spezialfall der allgemeinen Annahme des Presumed Influence ist der Third-Person-Effekt, bei dem die bei anderen angenommene Medienwirkung ins Verhältnis zu der auf sich selbst vermuteten Wirkung gesetzt wird. Diese Wahrnehmungsdifferenz kann sich u. a. auf die Regulierungsbereitschaft auswirken (im Überblick Feng \& Guo, 2012; Naab, 2013). Allerdings erscheint für das vorliegende Erkenntnisinteresse die Relativierung der vermuteten Medienwirkung auf andere an der auf die eigene Person konzeptuell nicht begründet. 
et al., 2001; Price et al., 1998; Rojas et al., 1996; für abweichende Ergebnisse Thompson, 1995; Youn et al., 2000).

Je nach thematischem Gegenstand der jeweiligen Studien nehmen ForscherInnen weitere psychografische Konstrukte in ihre Analyse auf. Unter anderem zeigen Studien mit moralisch aufgeladenen Themen einen Einfluss der Religiosität der Befragten (Golan, 2002; Gunther, 1995; Gunther \& Hwa, 1996; Hoffner et al., 1999; Price et al., 1998; Tsfati \& Cohen, 2003; Youn et al., 2000). Dies spiegelt sich auch im Einfluss des themenspezifischen Involvements (im Überblick Naab, 2013).

Mit dem Bildungsniveau steigen politische Toleranz und positive Einstellungen gegenüber der Meinungsfreiheit (Bernhard \& Dohle, 2014; Lambe, 2002). Allerdings finden nicht alle Studien Effekte (Gunther, 1995; Lee \& Tamborini, 2005; Youn et al., 2000). Tendenziell scheinen Frauen eher zu Regulierung zu neigen als Männer (Bernhard \& Dohle, 2014; Gunther, 1995; Hoffner \& Buchanan, 2002; Lee \& Tamborini, 2005; Lo \& Wei, 2002; McLeod et al., 2001; Suedfeld et al., 1994; Youn et al., 2000; Zhao \& Cai, 2008). Ältere sprechen sich häufiger für Beschränkungen aus (Bernhard \& Dohle, 2014; Dohle \& Bernhard, 2013; Huh et al., 2006).

\section{2 Übertragbarkeit auf soziale Medien}

Die aufgeführten Erkenntnisse wurden bislang kaum auf die Sanktionsbereitschaft gegenüber Botschaften in sozialen Medien übertragen. Dies scheint dringend geboten, denn es gibt Hinweise, dass die Regulierungsabsichten vom Kommunikationskanal abhängen. Hurwitz und Mondak (2002) unterscheiden zwischen „discriminatory intolerance“, also einer Ablehnung von Äußerungen mit bestimmten Inhalten, und „generic intolerance“, welche eine Ablehnung gegenüber bestimmten kommunikativen Akten unabhängig vom Kommunikationsinhalt meint. Studien, in denen Formen der generischen Intoleranz untersucht werden, sind selten und entbehren bislang einer systematischen Gegenüberstellung von Kommunikationskanälen. Überwiegend beschränken sich die Studien darauf, die Toleranz von verschiedenen Formen nicht-medialer oder traditioneller massenmedialer Ausdrucksformen abzufragen (Davis, 1990; International Social Survey Programme, 2012; Lambe, 2002). Bernhard und Dohle (2014) zeigen zusätzlich, dass JournalistInnen sich etwas mehr für Beschränkungen von Internetinhalten aussprechen als von Print- und Fernsehinhalten. Arbeiten sie für Online-Medien, halten sie Restriktionen allerdings für weniger wichtig. Die Erwartungen von Laien an OnlineKommunikation und die damit verbundenen Regulierungsabsichten sind kaum erforscht. Lediglich Trepte et al. (2008) stellen fest, dass die Qualitätsansprüche an Weblogs geringer sind als an Tageszeitungen. Eine Übertragung der bestehenden Erkenntnisse über Einflussfaktoren auf die Sanktionsbereitschaft gegenüber Botschaften in traditionellen Medien auf soziale Medien ist also nicht ohne Weiteres möglich und bedarf einer empirischen Prüfung.

\section{Hypothesen}

Aus der Forschung zu Einflussfaktoren auf die Zustimmung zu traditioneller Regulierung werden die folgenden Hypothesen über Determinanten des Sanktionsbedarfs aus Nutzersicht in den sozialen Medien abgeleitet.

H1: Je gefährlicher ein Kommunikator eingeschätzt wird, desto größer ist der empfundene Sanktionsbedarf. 
H2a: Je stärker die vermutete Wirkung eines Medieninhalts auf andere, desto größer ist der empfundene Sanktionsbedarf.

H2b: Je stärker die vermutete Wirkung eines Medieninhalts auf die eigene Person, desto größer ist der empfundene Sanktionsbedarf.

H3: Je umfangreicher die eigene Nutzung des Mediums, in dem ein Inbalt publiziert wird, desto geringer ist der empfundene Sanktionsbedarf.

H4: Je größer die Zustimmung zu Meinungsfreibeit im Allgemeinen, desto geringer ist der empfundene Sanktionsbedarf.

H5: Je konservativer die politische Orientierung, desto größer ist der empfundene Sanktionsbedarf.

H6: Männer empfinden einen geringeren Sanktionsbedarf als Frauen.

H7: Ältere empfinden einen größeren Sanktionsbedarf als Jüngere.

H8: Höber Gebildete empfinden einen geringeren Sanktionsbedarf als niedriger Gebildete.

In Hinblick auf den gewählten Stimulus der Untersuchung, eine Facebook-Gruppe der Scientology-Organisation (ausführlich siehe unten), wird außerdem postuliert:

H9: Stärker religiöse Menschen empfinden einen größeren Sanktionsbedarf als weniger religiöse Menschen.

Über diese Vermutungen hinaus sind Zusammenhänge zwischen mehreren Prädiktoren anzunehmen, die zu indirekten Effekten führen könnten. Die Literatur zeigt, dass Frauen, Ältere, niedriger Gebildete und politisch konservativ Orientierte zu einer höheren Gefahrenwahrnehmung neigen (Goodwin, Willson, Stanley \& Gaines, 2005; McLaren, 2003; Stevens et al., 2011; Stupi et al., 2014). Ältere und Frauen zeigen eher ablehnende Einstellungen gegenüber der Meinungsfreiheit im Allgemeinen (Lambe \& Reineke, 2009; Marcus et al., 1995). Studien unterstützen eine Verbindung zwischen Bildungsniveau und der Unterstützung für Bürgerrechte, politischer Toleranz, Offenheit gegenüber Fremden und einer Zustimmung zu post-materialistischen Werten wie Meinungsfreiheit (Lambe \& Reineke, 2009; McLeod, Sotirovic, Voakes, Guo \& Huang, 1998; Searing, Crewe \& Conover, 2007). Konservative lehnen Meinungsfreiheit eher ab (Lambe, 2002; McLeod et al., 1998; für abweichende Ergebnisse Lambe, 2008). ${ }^{2}$ Es ergibt sich das Modell in Abbildung 1.

\section{Methode}

\subsection{Design}

In einer standardisierten Online-Befragung wurde den Befragten ein Screenshot einer fiktiven Facebook-Gruppe „Scientology Deutschland“ vorgelegt, die für die Verbrei-

2 Darüber hinaus werden Korrelationen zwischen folgenden soziodemografischen und psychografischen Konstrukten eingefügt: Bildung und Alter, Bildung und Geschlecht, politische Orientierung und Bildung, politische Orientierung und Alter, politische Orientierung und Geschlecht, Religiosität und politische Orientierung, Religiosität und Bildung, Religiosität und Alter, Religiosität und Geschlecht, Alter und Facebook-Nutzungshäufigkeit, Bildung und Facebook-Nutzungshäufigkeit. Außerdem werden die Fehlerterme folgender Konstrukte korreliert: Eingeschätztes Gefahrenpotenzial und vermutete Wirkung auf die eigene Person und auf andere, vermutete Wirkung auf die eigene Person und vermutete Wirkung auf andere. 


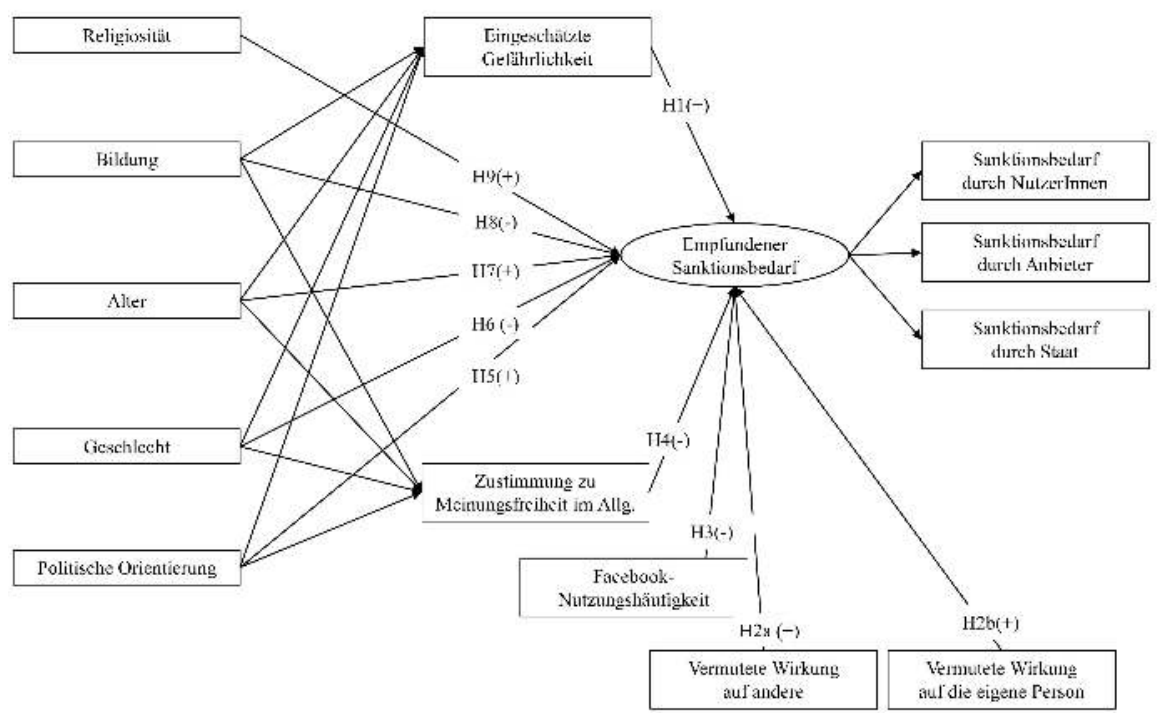

tung der Ziele der Scientology-Organisation eintritt. Soziale Netzwerke gehören zu den sozialen Medien-Angeboten mit weiter Verbreitung, und Facebook stellt den wichtigsten Anbieter dar (Duggan, Ellison, Lampe, Lenhart \& Madden, 2015; van Eimeren \& Frees, 2014). Damit beschränkt sich die Studie auf den empfundenen Sanktionsbedarf eines Facebook-Inhalts. Sie konzentriert sich auf die NutzerInnen dieser Plattform, weil diese mit den dort angebotenen Funktionalitäten vertraut sind und eine angemessene Sanktionierung auf der Plattform für sie relevanter sein dürfte als für Facebook-NichtNutzerInnen.

Die Gestaltung der Gruppenseite erfolgte in Orientierung an existierenden Facebook-Auftritten von Scientology-Anhängergruppen. Die Befragung legte einen konkreten Inhalt vor, dessen Regulierungsnotwendigkeit beurteilt werden sollte, weil Studien zeigen, dass die allgemeine Zustimmung zu Meinungsfreiheit von der Zustimmung zur Zulässigkeit konkreter Inhalte abweicht, wobei letztere geringer ausfällt (Peffley, Knigge \& Hurwitz, 2001). Die Befragten sahen lediglich die Gruppen-Startseite und keine Diskussionen oder Pinnwand-Einträge, weil nicht der empfundene Sanktionsbedarf konkreter Äußerungen, die innerhalb einer Kommunikatorgruppe sehr unterschiedlich ausfallen können, beurteilt werden sollte, sondern die Toleranz gegenüber einem Kommunikator insgesamt. Der Stimulus enthielt keine Inhalte, die nach deutschem Recht die Grenzen der Meinungsfreiheit überschreiten würden, so dass tatsächlich lediglich die grundsätzliche Intoleranz gegenüber potenziellen Äußerungen der Kommunikatorgruppe für die eingeschätzte Sanktionsnotwendigkeit relevant sein dürfte. Die Glaubensgemeinschaft Scientology wurde als Beispiel gewählt, weil sie in Deutschland umstritten ist. Die Scientology-Organisation wird vom Verfassungsschutz 
überwacht (Bundesministerium des Innern, 2013), allerdings steht sie weniger im Fokus gesellschaftlicher Debatten über die Zulässigkeit von Meinungsäußerungen als beispielsweise ausländerfeindliche oder religiös-fundamentalistische Medienbotschaften. Deshalb lässt sie vermutlich mehr Varianz in der Einschätzung des Gefahrenpotenzials und der Regulierungsnotwendigkeit zu und ruft eventuell weniger sozial erwünschte Antworten hervor.

\subsection{Operationalisierung}

Empfundener Sanktionsbedarf. In neun Items wurden mögliche negative soziale, technische und rechtliche Sanktionen gegenüber der Facebook-Gruppe beschrieben, die NutzerInnen selbst, der Plattformbetreiber Facebook oder der deutsche Staat ausführen können (Anhang 1). Die Befragten wurden um ihre Einschätzungen gebeten, ob sie auf die vorgelegte Facebook-Gruppe „Scientology Deutschland“ mit den genannten Sanktionen reagieren würden bzw. ob sie die Sanktionen von anderen Stellen erwarten würden (dichotom kodiert: „würde ich (nicht) tun“ bzw. „sollte Facebook (nicht) tun“ bzw. „sollte der Staat (nicht) tun“). Der Pretest zeigte, dass die ProbandInnen nur dichotome Antworten als angemessen empfanden, weil man eine Sanktion entweder ausführt oder nicht.

Theoriegeleitet wurden die Items zu Mittelwertindizes des Sanktionsbedarfs durch die drei Akteursgruppen NutzerInnen $(\mathrm{MW}=0,06 ; \mathrm{SD}=0,18 ; \alpha=.737)$, Facebook $(\mathrm{MW}=0,25 ; \mathrm{SD}=0,38 ; \alpha=.729)$ und Staat $(\mathrm{MW}=0,14 ; \mathrm{SD}=0,29 ; \alpha=.620)$ zusammengefasst. Die Analyse zeigte, dass Items zur Vorabprüfung der Facebook-Inhalte durch Facebook und den Staat nicht zur internen Konsistenz der Skalen beitrugen. Sie wurden deshalb aus der Analyse ausgeschlossen. Die Vorabprüfung stellt ein für den Staat verbotenes und für einen Plattformbetreiber schwer umsetzbares Verfahren dar. Eventuell wird eine Vorabprüfung auch von den Befragten nicht als Sanktion empfunden, weil sie keine Einschränkung der Inhalte nach sich ziehen muss.

Eingeschätzte Gefäbrlichkeit. Die Befragten gaben Auskunft über ihre Einschätzung der Gefahr durch Scientology und die gezeigte Facebook-Gruppe (jeweils mit den vier Items gefährlich, schädlich, schlecht, unnötig; semantisches Differential von $1=$ „nicht gefährlich“ bis $5=$,gefährlich“). Die acht Items wurden zu einem Mittelwertindex aggregiert $(\mathrm{MW}=4,02 ; \mathrm{SD}=0,63 ; \alpha=.834)$.

Vermutete Wirkung. Die vermutete Wirkung wurde erfragt, die die ScientologyFacebook-Gruppe auf eigenes Wissen, Glaubensausübung, Wertvorstellungen und Internetnutzung (z. B. für die Suche nach weiteren Informationen oder eine Kontaktaufnahme zur Scientology-Organisation) der Befragten hat (Skala von $1=$ „sehr gering“ bis $7=$ „sehr hoch“; MW = 1,51; SD =0,76; $\alpha=.674)$. Hinsichtlich der gleichen Dimensionen wurde die vermutete Wirkung auf andere erfragt $(\mathrm{MW}=2,63 ; \mathrm{SD}=1,04 ; \alpha=.794)$.

Facebook-Nutzungshäufigkeit, erhoben von $0=$ "nie“ bis $7=$ "mehrmals am Tag“ $(\mathrm{MW}=6,15 ; \mathrm{SD}=1,26)$. Aufgrund der Schiefverteilung wurde die Variable dichotomisiert; 21,1 Prozent nutzen die Plattform höchstens mehrmals die Woche.

Zustimmung zu Meinungsfreibeit im Allgemeinen. erfragt in Anlehnung an Miklikowska (2011; fünf Items, z. B. „Jede/r sollte die Möglichkeit haben, seine Meinung öffentlich zu äußern, unabhängig davon, wie kontrovers diese ist“; Skala von $1=$ „trifft

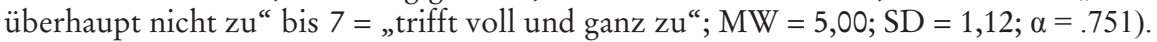

Politische Orientierung. Die Befragten machten Angaben über ihre politische Einstellung (vgl. Gesis, 2014; Skala von $1=$ „links“ bis $10=$ „rechts“; $M W=4,61$; $S D=1,55$ ).

Religiosität. Die Religiosität der Befragten wurde mit einer Skala zur persönlichen Häufigkeit religiöser Praktiken und Erfahrungen gemessen (CRS-5 von Huber \& 
Huber, 2012; fünf Items; Skala von $1=$ „nie“ bis $7=$,sehr häufig“; $\mathrm{MW}=2,96$; $\mathrm{SD}=$ $1,46 ; \alpha=.881)$. Aufgrund der Schiefverteilung wurde die Variable nach Mediansplit dichotomisiert. Kein/e Befragte/r gehört der Scientology-Organisation an.

\subsection{Stichprobe}

Im Juni 2014 wurden deutschsprachige Facebook-User befragt. Um ProbandInnen mit vielfältigen sozialen Hintergründen und variierenden Einstellungen gegenüber der Scientology-Organisation und dem Schutz von Grundrechten zu erreichen, erfolgte die Rekrutierung über unterschiedlichste Facebook-Gruppen. Es wurden die AdministratorInnen einer Vielzahl von Gruppen kontaktiert, die nach Gruppentitel und -beschreibung religionsnah bzw. -fern waren, verschiedene politische Orientierungen teilten, auf jüngere bzw. ältere und höher bzw. niedriger gebildete Mitglieder abzielten. ${ }^{3}$ Von denen, die auf den Link zur Befragung geklickt hatten $(\mathrm{N}=370)$, nahmen 84 Prozent teil und 72 Prozent beendeten die Befragung vollständig. Dies sind im Vergleich zu üblichen Abbruchquoten gute Werte (Macias, Springston, Weaver \& Neustifter, 2008). Die finale Stichprobe derer, die die Befragung vollständig ausfüllten, umfasst 265 Personen. 62 Prozent der Befragten sind weiblich. Der Altersdurchschnitt liegt bei 26,6 Jahren $(\mathrm{SD}=8,20)$. Dass sich Jüngere verstärkt privaten Online-Communities zuwenden, spiegelt sich deutlich in diesem Ergebnis. In der Gruppe der unter 30-Jährigen ist die Nutzung sozialer Netzwerke weit verbreitet. Bei den Älteren nimmt die Nutzung deutlich ab (von $74 \%$ auf $37 \%$, van Eimeren \& Frees, 2014). Für die Auswertungen wurde die Variable aufgrund der Abweichung von der Normalverteilung dichotomisiert; 51,7 Prozent sind unter 25 Jahren.

86 Prozent der Befragten haben Fachhochschulreife oder einen höheren Schulabschluss. Andere Studien zeigen demografische Verteilungen der Facebook-Nutzerschaft, die etwa 50 Prozent AkademikerInnen und weitere 18 Prozent AbiturientInnen ausweisen (Kirch, 2013). Das vorliegende Sample ist folglich etwas zugunsten höher Gebildeter verzerrt. Das spiegelt die grundsätzliche Schwierigkeit, in selbstselektiven Online-Befragungen niedrig Gebildete zu motivieren. Für die Auswertungen wurde die Variable dichotomisiert; 36,6 Prozent haben ein Studium abgeschlossen.

In ihrer Selbsteinstufung der eigenen politischen Orientierung von links bis rechts weicht die Stichprobe nur geringfügig nach links von der deutschen Gesamtbevölkerung ab (0,43 Skalenpunkte Differenz; Gesis, 2014). Außerdem ist sie geringfügig weniger religiös (0,79 Skalenpunkte Differenz beim Item „Wie häufig beten Sie?“; Gesis, 2012). Diese Abweichungen dürften durch das jüngere Alter der Facebook-NutzerInnen zu erklären sein.

\section{Ergebnisse}

Die Hypothesen wurden mit Hilfe eines Strukturgleichungsmodells zur Erklärung des von NutzerInnen empfundenen Sanktionsbedarfs der Facebook-Gruppe geprüft. Das Modell zeigt eine gute Anpassung an die Daten $(n=264 ; p=.092 ; \mathrm{CMIN}(43)=55,766$; RMSEA =0,034; PCLOSE =0,869; CFI =0,964; SRMR =0,050). Das Signifikanzniveau aller Effekte wurde mittels Bootstrapping ermittelt. Es wurden 2000 Bootstrap Samples und Bias-Corrected Confidence Intervals genutzt (Abb. 2). Das Modell zeigt signifi-

3 Von welchen Facebook-Gruppen letztlich tatsächlich ProbandInnen auf die Befragung aufmerksam wurden und den Link angeklickt haben, ist mit der verwendeten Befragungssoftware nicht nachvollziehbar. 
kante, direkte Effekte der Gefahreneinschätzung ( $(=.589 ; p \leq .001)$, des Bildungsniveaus $(ß=-.136 ; p=.027)$ und der Facebook-Nutzungshäufigkeit $(B=-.144 ; p=.023)$. Personen, die die Scientology-Kommunikation für gefährlich halten, halten Maßnahmen eher für angebracht. AkademikerInnen und Personen, die die Social Media-Plattform mindestens einmal täglich nutzen, stimmen einer Einschränkung der Inhalte eher nicht zu. $\mathrm{H} 1, \mathrm{H} 3$ und $\mathrm{H} 8$ können beibehalten werden.

Die weiteren Faktoren zeigen keinen signifikanten, direkten Einfluss. Keinen Einfluss haben die vermutete Wirkmächtigkeit auf andere $(\beta=.016 ; p=.824)$ und auf die eigene Person ( $(=.017 ; p=.785)$. Personen, die die Meinungsfreiheit generell als weniger wichtigen Wert erachten, zeigen sich, anders als erwartet, gegenüber der FacebookGruppe nicht weniger aufgeschlossen $(ß=-.076 ; p=.233)$. Gleiches gilt für politisch Konservative $(B=-.075 ; p=.301)$. Die häufig in der Literatur thematisierten Effekte, dass Frauen $(B=.032 ; p=.638)$ und Ältere $(B=.025 ; p=.705)$ eher zu Einschränkungen neigen, bestätigen sich nicht. Auch stärker religiöse Menschen schätzen den Sanktionsbedarf der Scientology-Organisation nicht größer ein $(B=-.160 ; p=840)$. H2a, H2b, $\mathrm{H} 4, \mathrm{H} 5, \mathrm{H} 6, \mathrm{H} 7$ und $\mathrm{H} 9$ werden abgelehnt. Allerdings gibt es geringe, signifikante indirekte Effekte der politischen Orientierung $(B=.087 ; p=.009)$ sowie des Alters $(B=$. 097; $p=.019)$, die der postulierten Richtung entsprechen. Die anderen Faktoren zeigen keine signifikanten indirekten Effekte. ${ }^{4}$

\section{Diskussion}

Es wurde erstmals gemessen, ob Nutzende einen Facebook-Inhalt als sanktionsbedürftig empfinden. Außerdem wurden potenzielle Einflussfaktoren aus der Literatur zu traditionellen Massenmedien hergeleitet und ihre Bedeutsamkeit im Kontext sozialer Netzwerke empirisch geprüft.

Die Operationalisierung der Sanktionsmittel orientierte sich an den Optionen, die Nutzenden in Facebook-Gruppen zur Verfügung stehen, und denen, die sie von Autoritäten erwarten könnten, die über technische oder rechtliche Regulierungsmöglichkeiten verfügen. Insgesamt sind die Zustimmungswerte zu den einschränkenden Sanktionsmitteln der drei Akteursgruppen eher niedrig. Auch wenn die absoluten Werte ob der noch zu diskutierenden Stichprobengenerierung nur mit großer Vorsicht interpretiert werden dürfen, soll angemerkt werden, dass vor allem nutzerseitige Sanktionsmaßnahmen wenig Anklang bei den ProbandInnen finden. Am ehesten sehen diese Facebook in der Pflicht, Inhalte zu löschen oder NutzerInnen zu sperren. Es kann spekuliert werden, dass technische Maßnahmen als wirkstärker als nutzerseitige, soziale Sanktionen empfunden werden und als wahrscheinlicher als ein Eingreifen des Staates. Vier Erklärungsansätze für die geringen Sanktionswerte sollen diskutiert werden:

1) Möglicherweise wird das Scientology-Beispiel nicht negativ genug eingeschätzt. Dagegen spricht aber der Mittelwert des eingeschätzten Gefahrenpotenzials, der deutlich über dem Skalenmittel liegt.

4 Es bestätigt sich allerdings, dass Ältere und politisch konservativer Orientierte zu einer höheren Gefahreneinschätzung neigen sowie dass Ältere und Frauen Meinungsfreiheit weniger wertschätzen (vgl. Abb. 2). Außerdem erwiesen sich die Korrelationen zwischen folgenden Konstrukten bzw. Fehlertermen als signifikant (vgl. Fußn. 2): Bildung und Alter, Religiosität und Alter, Religiosität und Geschlecht, Alter und Facebook-Nutzungshäufigkeit, vermutete Wirkung auf die eigene Person und vermutete Wirkung auf andere. 
Abbildung 2: Empirisches Modell der Einflïsse auf den empfundenen Sanktionsbedarf

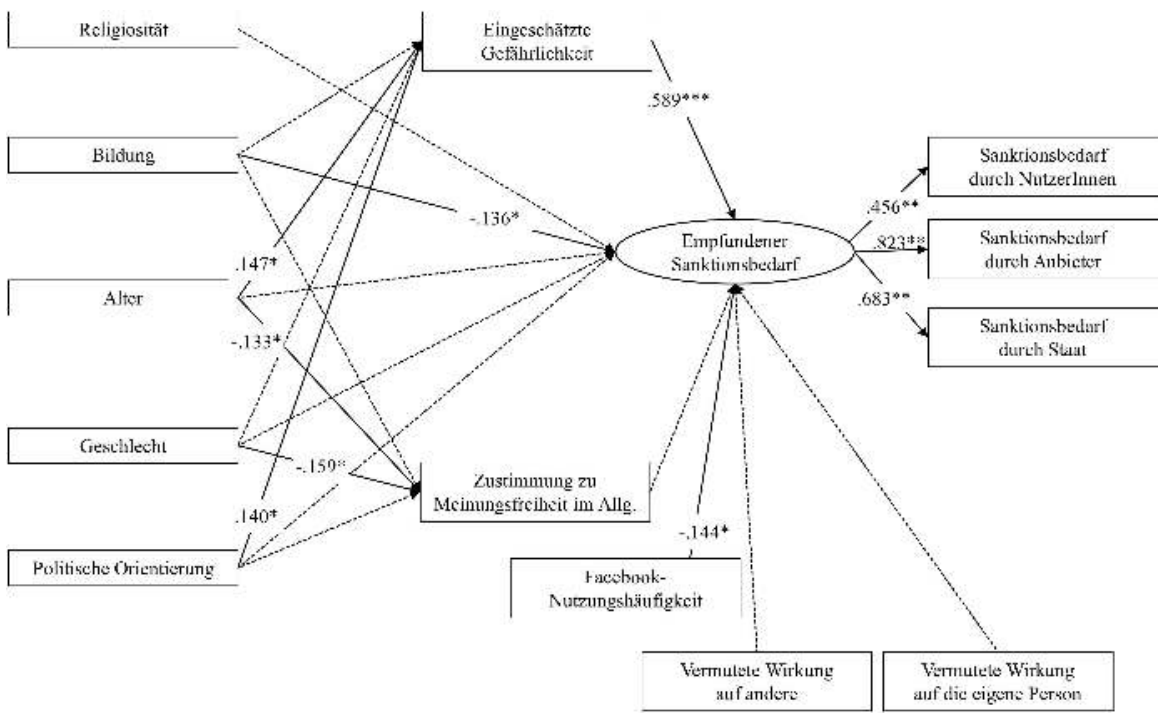

$\mathrm{n}=264 ; \mathrm{p}=.092 ; \mathrm{CMIN}(43)=55,766 ;$ RMSEA $=0,034 ; \mathrm{PCLOSE}=0,869 ; \mathrm{CFI}=0,964 ;$ SRMR $=$ 0,050 .

$* \mathrm{p} \leq .05 ; * \mathrm{p} \leq .01 ; * * \mathrm{p} \leq .001$.

Gestrichelte Pfeile zeigen nicht signifikante Pfade.

2) Eventuell wird Facebook-Inhalten insgesamt wenig Relevanz beigemessen. Dahin deuten auch die niedrigen Mittelwerte für die vermutete Wirkung auf andere und auf die eigene Person. Die Literatur zum Presumed Influence zeichnet kein klares Bild über den Einfluss der Mediengattung auf die Wirkvermutung, und soziale Medien wurden bislang wenig berücksichtigt (im Überblick Naab, 2013), so dass weitere Forschung zeigen muss, ob der Sanktionsbedarf aus Nutzersicht von der vermeintlichen Bedeutsamkeit von Social Media-Inhalten abhängt.

3) Eventuell sehen die Befragten sich trotz ihrer in den sozialen Medien neu gewonnenen Sanktionsmöglichkeiten nicht selbst in der Verantwortung, zu regulieren und sich überhaupt mit Limitationsoptionen auseinanderzusetzen, selbst wenn diese von Autoritäten ausgeführt würden. Eine solche grundsätzliche Lethargie zeigt sich auch in Studien zu Nutzerkommentaren. Sogar wenn User in eine Diskussion involviert sind, ist doch eine Regulierung unangemessener Kommentare Anderer durch die User selten (z. B. Ziegele, Breiner \& Quiring, 2014). Dies kann einerseits ob der oben diskutierten positiven Folgen nutzerseitiger Sanktionierung bedauert werden. Andererseits sind Sanktionen durch Laien nicht demokratisch legitimiert und orientieren sich nicht notwendigerweise an grundgesetzlich definierten Wertmaßstäben. Ein geringes nutzerseitiges Eingreifen kann deshalb auch positiv gedeutet werden als Hinweis auf eine hohe Toleranz in der Leserschaft. Aus dieser Perspektive könnten die absoluten Werte zur Sanktionsbereitschaft Anlass zur Sorge geben: Die vorgelegte, fiktive Facebook-Gruppenseite enthielt keine rechtlich bedenklichen Inhalte. Dennoch fanden 39 Prozent der Befragten mindestens eines der Sanktionsmittel angemessen. 
4) Letztlich muss ein methodisches Defizit bedacht werden: Es handelte sich um die Messung des hypothetischen Sanktionsbedarfs gegenüber einer fiktiven FacebookGruppe. Dies ist zwar ein durchaus übliches Vorgehen in zahlreichen Studien, dennoch kann es die wahrgenommene Notwendigkeit einer Regulierung für die Befragten eingeschränkt haben. Auch die Tatsache, dass sie lediglich einen Screenshot einer Facebook-Gruppenseite sahen, ohne deren weitere Einbindung in das soziale Netzwerk, mag die wahrgenommene Dringlichkeit des eigenen Vorgehens reduziert haben.

Stärkster Einflussfaktor auf die Zustimmung zu Einschränkungen der Facebook-Gruppe ist die vermeintliche Gefahr, die von der Scientology-Organisation und ihrer Facebook-Gruppe ausgeht. Das Ausmaß der vermuteten Wirkung auf die eigene Person und auf andere spielt keine Rolle. Offensichtlich ist es für hypothetische, limitierende Reaktionen irrelevant, ob eine große Wirkung auf verschiedene Alltagsbereiche zu erwarten ist, solange der Kommunikator als solcher als bedrohlich empfunden wird. Dieses Ergebnis bestätigt Befunde aus Studien zu Presumed Influence, die zeigen, dass die Evaluation der Botschaft einen stärkeren (oder alleinigen) Einfluss hat als die Wirkvermutung (Bernhard \& Dohle, 2014) und die wahrgenommene Unangemessenheit einer Botschaft nicht mit ihrer vermuteten Wirkung korrelieren muss (Lambe \& McLeod, 2005; McLeod et al., 2001; Paek et al., 2008; Salwen \& Dupagne, 1999).

Eine hohe Facebook-Nutzungshäufigkeit verringert den Wunsch nach Sanktionen gegenüber der problematischen Facebook-Gruppe. Anders als die ProbandInnen der Studie von Trepte und Kollegen (2008) zu Weblogs sprechen sich die befragten Facebook-NutzerInnen nicht für mehr Kontrolle aus. Eventuell betrachten sie Facebook als Plattform mit vorrangig vielfältigem Angebot, bei dem die Breite der Inhalte wichtiger ist als ihre Qualität.

Die grundsätzliche Wertschätzung der Meinungsfreiheit hat keinen Einfluss auf den empfundenen Sanktionsbedarf. Die Hypothese basierte auf Befunden, dass die allgemeine Zustimmung zu einem Grundrecht auch seine Auslegung im speziellen Anwendungsfall bestimmt. Allerdings betonen einige AutorInnen Inkonsistenzen zwischen der Zustimmung im Abstrakten und gegenüber speziellen, als anstößig empfundenen Äußerungen. Peffley und Kollegen (2001) weisen darauf hin, dass die Entscheidung über die Unterstützung von Restriktionen in konkreten Fällen vor dem Hintergrund konkurrierender Werte getroffen werden muss. Wenn Menschen sich der Tatsache bewusst werden, dass Meinungsfreiheit mit anderen Rechten, wie beispielsweise dem Schutz der Jugend, kollidiert, sinkt ihre Unterstützung erkennbar. Dies könnte sich in den Daten spiegeln, und die Facebook-Nutzenden messen der Meinungsfreiheit eventuell grundsätzlich mehr oder weniger Bedeutung bei, was aber im konkreten Fall der (fiktiven) Kommunikation der Scientology-Organisation in den Hintergrund tritt.

Alter und politische Orientierung der Befragten wirken lediglich vermittelt über die Gefahreneinschätzung auf den Wunsch nach Einschränkungen der Facebook-Gruppe. Dies betont, dass diese soziodemografischen und psychografischen Gruppen nicht grundsätzlich weniger tolerant sind, sondern eine andere Haltung gegenüber der zur Diskussion stehenden Kommunikatorgruppe einnehmen. Ein höheres Bildungsniveau reduziert den Ruf nach Sanktionen; dieser Effekt wurde bereits vielfach für traditionelle Medien bestätigt. Der Einfluss ist unabhängig von der Gefahreneinschätzung. Höher Gebildete haben also keine andere Einstellung zur Scientology-Organisation oder ihren kommunikativen Akten auf Facebook; vielmehr ist zu vermuten, dass sie sich im Laufe ihrer Ausbildung häufiger mit der Bedeutung von Grundrechten wie Meinungsfreiheit, der Toleranz anderer Einstellungen und verschiedenen Formen der Konfliktlösung aus- 
einandergesetzt haben und deshalb weniger zu Restriktionen neigen. Die Religiosität der Befragten spielt keine Rolle. Vermutlich tangiert die Kommunikation der ScientologyOrganisation, die in Deutschland nicht als Religionsgemeinschaft anerkannt ist und nur eine geringe Verbreitung hat, nicht die persönlichen religionsbezogenen Werte der Befragten.

Die Studie bietet einen ersten Ansatzpunkt zur empirischen Untersuchung des Sanktionsbedarfs nach Einschätzung durch Laien in sozialen Netzwerken. Die Aussagekraft der Ergebnisse ist allerdings durch mehrere Faktoren reduziert. Zum einen arbeitet die Studie mit einer fiktiven Medienbotschaft und untersucht hypothetische Sanktionen. Zwar gehen die Möglichkeiten der technischen Beobachtung von Nutzerverhalten in Online-Medien weit über die in traditionellen Medien hinaus. Aktionen wie das Melden, Bewerten, Weiterverbreiten konkreter Inhalte, die nicht von den Forschenden erstellt wurden, sind dennoch schwer zu erfassen - zumal wenn diese Aktionen mit Personendaten und individuellen Einschätzungen zu den Medienbotschaften verknüpft werden sollen. Die Studie bedient sich deshalb einer hypothetischen Frageformulierung, wie diese in vielen Studien zu Regulierungseinstellungen üblich ist. Hinzu kommt, dass die im Fragebogen vorgeschlagenen Sanktionsmittel nicht alle gleichermaßen wahrscheinlich sind, zumal die fiktive Facebook-Gruppe keine rechtswidrigen Inhalte enthielt. Damit sollte den Befragten die Möglichkeit gegeben werden, aus ihrer Sicht angemessene Mittel anzugeben (vgl. Lambe, 2002). Die Fragen zu klären, in welchem Umfang Laien über Sanktionsmittel informiert sind, ob sie sich eventuell gegen ein Mittel aussprechen, weil sie wissen, dass es nicht angewendet wird oder sie es für nicht zulässig halten, und welche Motive Laien mit den jeweiligen Sanktionen verfolgen, bleibt weiteren Studien vorbehalten.

Des Weiteren konzentriert sich das Design auf eine Facebook-Gruppe und untersucht ausschließlich Facebook-NutzerInnen. Problematische Botschaften sind ebenso in anderen Online Communities und Social Media-Angeboten zu finden. Eine Übertragbarkeit der Ergebnisse ist jedoch nicht gewährleistet, weil sich sowohl die Ansprüche der NutzerInnen an die verschiedenen Plattformen unterscheiden als auch die Betreiber unterschiedliche Mittel zur Sanktionierung durch die NutzerInnen anbieten und selbst in unterschiedlichem Maße aktiv werden (FSM, 2015).

Die Beschränkung auf eine spezielle Kommunikatorgruppe erschwert die Übertragbarkeit auf andere Medienbotschaften. Die Scientology-Gruppe wird zwar von den Befragten als bedrohlich wahrgenommen, gehört aber nicht zu den Randgruppen, mit denen Mediennutzende im Alltag häufig konfrontiert werden. Deshalb bestehen gegenüber der Glaubensgemeinschaft und ihren kommunikativen Bemühungen vermutlich weniger Voreinstellungen, die sich im empfundenen Sanktionsbedarf niederschlagen könnten. Möglicherweise zeigen sich die postulierten Effekte bei Studien mit Kommunikatorgruppen mit größerer gesellschaftlicher Präsenz deutlicher. Sollten sich andere Einflussgrößen als im vorgelegten Modell als relevant erweisen, weist dies auf die Notwendigkeit hin, die gesellschaftliche Präsenz zu berücksichtigen.

Die Stichprobe der Untersuchung wurde nicht zufällig gewählt. Zwar wurde breit in Facebook rekrutiert, dennoch konnten Verzerrungen nicht verhindert werden. Dies schränkt sowohl die Verlässlichkeit der absoluten Werte des Sanktionsbedarfs als auch der gefundenen Zusammenhänge ein. Eine Replikation mit repräsentativer Stichprobe ist deshalb unumgänglich. Vor allem sind die Befragten höher gebildet als die tatsächliche Facebook-Nutzerschaft. Bemerkenswert ist allerdings, dass dennoch ein Einfluss des Bildungsniveaus festgestellt werden konnte. Der Ruf nach Sanktionen unterscheidet sich zwischen AkademikerInnen und Nicht-AkademikerInnen in der postulierten Richtung. 
Es ist zu erwarten, dass der Effekt noch deutlicher hervortritt, wenn weitere Personen niedrigerer Bildungsgrade in die Analyse eingeschlossen werden.

Das Modell ist nicht erschöpfend. Die Studie konzentrierte sich darauf, die Erklärkraft von Faktoren zu untersuchen, die aus der Forschung zu traditionellen Medien bekannt sind. Zum einen müssen weitere Faktoren geprüft werden, die sich als wirksam erwiesen haben (z. B. Paternalismus, McLeod et al., 1997, 2001; Sun, Pan \& Shen, 2008). Zum anderen muss das Modell in zukünftigen Studien ergänzt werden um Faktoren, die spezifisch für soziale Medien sind. Zu denken ist an die Einstellung zu Internetregulierung (vgl. BBC World Service, 2010; Kull et al., 2008). Diese kann von der allgemeinen Zustimmung zu Meinungsfreiheit abweichen, wenn NutzerInnen beispielsweise die Möglichkeiten zur Gegenrede gegen unliebsame Botschaften als Argument gegen Regulierung von Internetinhalten vorbringen (z. B. United Nations General Assembly, 2011). Auch besteht unter Laien unterschiedliches Wissen über den tatsächlichen Umfang der Internetkontrolle (Stichwort: Internet als vermeintlich rechtsfreier Raum). Weiterhin differieren vermutlich die Qualitätsansprüche, die an Botschaften in den sozialen Medien herangetragen werden, die wahrgenommene Glaubwürdigkeit der Inhalte und Professionalität der KommunikatorInnen (z. B. Banning \& Sweetser, 2007; im Überblick Naab, 2013), was ebenfalls die Notwendigkeit von Sanktionen beeinflussen kann. Die NutzerInnen können ihr Mit-Publikum und dessen Größe häufig nur schätzen. Diese Schätzung könnte über die Bedeutungszuweisung an die kommunizierten Inhalte und die Wirkvermutung den Sanktionsbedarf beeinflussen (vgl. Hartmann \& Dohle, 2005). Letztlich sind Studien, die einen Vergleich von Sanktionen gegen Botschaften in verschiedenen Mediengattungen anstellen, notwendig.

\section{Fazit}

Das Manuskript gibt einen Einblick, welche Sanktionen Laien für angemessen halten gegen eine Kommunikatorgruppe, die sozial nicht vollständig toleriert ist. In sozialen Medien ergänzen erstmals nutzerseitige Feedbackmaßnahmen die Palette möglicher Sanktionen, die von Plattformbetreibern und dem Staat ausgeführt werden können. So nehmen Laien nicht nur durch die Produktion eigener Inhalte am öffentlichen Diskurs teil, sondern auch durch ihre Positionierung gegenüber den Kommunikaten anderer. Damit zeigt der Beitrag ein neues Forschungsfeld auf, in dessen Zentrum die Beurteilung und Sanktionierung von Inhalten durch Laien stehen. Fragen werden relevant unter anderem nach den Wertmaßstäben, die NutzerInnen bei der Beurteilung von Social MediaInhalten anlegen, nach der Übereinstimmung dieser Maßstäbe mit journalistischen Qualitätskriterien und demokratischen Ansprüchen an Meinungsfreiheit. Denn die meisten Social Media User haben keine institutionalisierte Ausbildung über die Bedeutung von Meinungsfreiheit und ihre Grenzen erhalten. Die Einschätzung von Laien über die Wirkmächtigkeit von nutzerseitigen, anbieterseitigen und rechtlichen Sanktionsmitteln stellt deshalb eine neue Facette der wissenschaftlichen Betrachtung der Regulierung neuer Medien dar. Die Ergebnisse verweisen außerdem auf die Wichtigkeit, systematisch Unterschiede in den Determinanten des Sanktionsbedarfs in traditionellen und neuen Mediengattungen zu untersuchen.

\section{Literatur}

Bahry, D., Boaz, C. \& Gordon, S. B. (1997). Tolerance, transition, and support for civil liberties in Russia. Comparative Political Studies, 30(4), 484-510. 
Banning, S. A. \& Sweetser, K. D. (2007). How much do they think it affects them and whom do they believe? Comparing the third-person effect and credibility of blogs and traditional media. Communication Quarterly, 55(4), 451-466.

BBC World Service. (2010). Four in five regard internet access as a fundamental right: Global poll. http://www.globescan.com/news_archives/bbc2010_internet/ [22.01.2016].

Bernhard, U. \& Dohle, M. (2014). Do even journalists support media restrictions? Presumed political media influences and the consequences. Journalism \& Mass Communication Quarterly, 91(2), 250-271.

Bruckman, A., Danis, C., Lampe, C., Sternberg, J. \& Waldron, C. (2006). Managing deviant behavior in online communities. In A. Henderson \& J. A. Euchner (Eds.), Proceedings of the CHI 2006 Conference on Human Factors in Computing Systems (S. 21-24). New York, NY: ACM.

Buchloh, S. (2003). Überlegungen zu einer Theorie der Zensur. Interessen - Formen - „Erfolgsfaktoren“. In W. R. Langenbucher (Hrsg.), Die Kommunikationsfreiheit der Gesellschaft. Die demokratischen Funktionen eines Grundrechts (S.112-135). Wiesbaden: Westdeutscher.

Bundesministerium des Innern (2013). Verfassungsschutzbericht 2013. http://www.verfassungsschutz.de/de/oeffentlichkeitsarbeit/publikationen/verfassungsschutzberichte [22.01.2016].

Crawford, K. \& Gillespie, T. (2014). What is a flag for? Social media reporting tools and the vocabulary of complaint. New Media E Society, published online before print. Verfügbar unter http://nms.sagepub.com/content/early/2014/07/15/1461444814543163.abstract [22.01.2016].

Davis, J. A. (1990). Attitudes towards free speech in six countries in the mid-1980: Australia, Austria, Great Britain, Italy, the United States and West Germany. European Sociological Review, 6(1), $1-14$

Dohle, M. \& Bernhard, U. (2013). Presumed online media influence and support for censorship: Results from a survey among German parliamentarians. International Journal of Public Opinion Research, 26(2), 256-268.

Döring, N. (2001). Belohnungen und Bestrafungen im Netz: Verhaltenskontrolle in Chat-Foren. Gruppendynamik und Organisationsberatung, 32(2), 109-143.

Duggan, M., Ellison, N. B., Lampe, C., Lenhart, A. \& Madden, M. (2015, 9. Januar). Social media update 2014. http://www.pewinternet.org/2015/01/09/social-media-update-2014/ [22.01. 2016].

Feng, G. C. \& Guo, S. Z. (2012). Support for censorship: A multilevel meta-analysis of the thirdperson effect. Communication Reports, 25(1), 40-50.

FSM, Freiwillige Selbstkontrolle Multimedia-Diensteanbieter e. V. (2013). Selbstverpflichtungen der Wirtschaft. Social Communities. http://www.fsm.de/selbstverpflichtungen/social-commu nities [22.01.2016].

Gesis - Leibniz-Institut für Sozialwissenschaften (2012). Allbus, Allgemeine Bevölkerungsumfrage der Sozialwissenschaften. http://www.gesis.org/allbus/studienprofile/2012/ [22.01.2016].

Gesis - Leibniz-Institut für Sozialwissenschaften (2014). Allbus, Allgemeine Bevölkerungsumfrage der Sozialwissenschaften. http://www.gesis.org/allbus/studienprofile/2014/ [22.01.2016].

Gibbs, J. P. (1981). Norms, deviance, and social control. Conceputal matters. New York, NY: Elsevier.

Gibson, J. L. \& Bingham, R. D. (1983). Elite tolerance of Nazi rights. American Politics Research, 11(4), 403-428.

Gibson, J. L. (2006). Enigmas of intolerance: Fifty years after Stouffer's communism, conformity, and civil liberties. Perspectives on Politics, 4(1), 21-34.

Golan, G. (2002). Religiosity and the third-person effect. Journal of Media and Religion, 1(2), 105120.

Goodman, E. (2013). Online comment moderation: emerging best practices. http://www.wanifra.org/reports/2013/10/04/online-comment-moderation-emerging-best-practices [22.01.2016].

Goodwin, R., Willson, M., Stanley, G. \& Gaines, S. (2005). Terror threat perception and its consequences in contemporary Britain. British Journal of Psychology, 96(4), 389-406.

Gunther, A. C. (1995). Overrating the X-rating: The third-person perception and support for censorship of pornography. Journal of Communication, 45(1), 27-38. 
Gunther, A. C. \& Hwa, A. P. (1996). Public perceptions of television influence and opinions about censorship in Singapore. International Journal of Public Opinion Research, 8(3), 248-265.

Gurzick, D., White, K. F., Lutters, W. G. \& Boot, L. (2009). A view from Mount Olympus: The impact of activity tracking tools on the character and practice of moderation. In S. Teasley, E. Havn, W. Prinz \& W. Lutters (Eds.), Proceedings of the ACM 2009 International Conference on Supporting Work Group (S. 361-369). New York, NY: ACM.

Hartmann, T. \& Dohle, M. (2005). Publikumsvorstellungen im Rezeptionsprozess. Publizistik, 50(3), 287-303.

Hoffner, C. \& Buchanan, M. (2002). Parents' responses to television violence: The third-person perception, parental mediation, and support for censorship. Media Psychology, 4(3), 231-252.

Hoffner, C., Buchanan, M., Anderson, J. D., Hubbs, L. A., Kamigaki, S. K., Kowalczyk, L., Pastorek, A., Plotkin, R. S. \& Silberg, K. J. (1999). Support for censorship of television violence: The role of the third-person effect and news exposure. Communication Research, 26(6), 726 742.

Huber, S. \& Huber, O. W. (2012). The centrality of religiosity scale (CRS). Religions, 3(4), 710-724.

Huh, J., Delorme, D. E. \& Reid, L. N. (2006). Perceived third-person effects and consumer attitudes on preventing and banning DTC advertising. Journal of Consumer Affairs, 40(1), 90-116.

Hurwitz, J. \& Mondak, J. J. (2002). Democratic principles, discrimination and political intolerance. British Journal of Political Science, 32(1), 93-118.

International Social Survey Programme (2012). International Social Survey Programme. http:// www.issp.org/ [22.01.2016].

Jugendschutz.net (2014). Jugendschutz im Internet. Ergebnisse der Recherchen und Kontrollen. http://www.jugendschutz.net/fileadmin/download/pdf/bericht2014.pdf [22.01.2016].

Kirch, N. (2013). Auswertung der Jahresumfrage Social Media Statistik. http://www.socialmediastatistik.de/auswertung-der-jahresumfrage-social-media-statistik/ [22.01.2016].

Kull, S., Ramsay, C., Weber, S., Lewis, E., Ciolek, M., Brouwer, M. \& Medoff, A. (2008). World public opinion and the universal declaration of human rights. http://worldpublicopinion.org/ pipa/pdf/dec08/WPO_UDHR_Dec08_rpt.pdf [22.01.2016].

Lambe, J. L. \& McLeod, D. M. (2005). Understanding third-person perception processes: Predicting perceived impact on self and others for multiple expressive contexts. Journal of Communication, 55(2), 277-291.

Lambe, J. L. \& Reineke, J. B. (2009). Public attitudes about government involvement in expressive controversies. Journal of Communication, 59(2), 225-242.

Lambe, J. L. (2002). Dimensions of censorship: Reconceptualizing public willingness to censor. Communication Law E Policy, 7(2), 187-235.

Lambe, J. L. (2004). Who wants to censor pornography and hate speech? Mass Communication $\mathcal{E}$ Society, 7(3), 279-299.

Lambe, J. L. (2008). The structure of censorship attitudes. Communication Law E Policy, 13(4), 485-506.

Lee, B. \& Tamborini, R. (2005). Third-person effect and internet pornography: The influence of collectivism and internet self-efficacy. Journal of Communication, 55(2), 292-310.

Lo, V.-h. \& Wei, R. (2002). Third-Person effect, gender, and pornography on the internet. Journal of Broadcasting E Electronic Media, 46(1), 13-33.

Macias, W., Springston, J. K., Weaver, R. A. L. \& Neustifter, B. (2008). A 13-year content analysis of survey methodology in communication related journals. Journal of Current Issues E Research in Advertising, 30(1), 79-94.

Marcus, G. E., Sullivan, J. L., Theiss-Morse, E. \& Wood, S. L. (1995). With malice toward some. How people make civil liberties judgments. Cambridge, UK: Cambridge University Press.

McLaren, L. M. (2003). Anti-immigrant prejudice in Europe: Contact, threat perception, and preferences for the exclusion of migrants. Social Forces, 81(3), 909-936.

McLeod, D. M., Detenber, B. H. \& Eveland, W. P. (2001). Behind the third-person effect: Differentiating perceptual processes for self and other. Journal of Communication, 51(4), 678-695.

McLeod, D. M., Eveland, W. P. \& Nathanson, A. I. (1997). Support for censorship of violent and misogynic rap lyrics: An analysis of the third-person effect. Communication Research, 24(2), 153-174. 
McLeod, J. M., Sotirovic, M., Voakes, P. S., Guo, Z. \& Huang, K.-Y. (1998). A model of public support for First Amendment rights. Communication Law E Policy, 3(4), 479-514.

Miklikowska, M. (2011). Democracy begins at home. Parenting, empathy, and adolencents' support for democratic values. Vasa: KTMP.

Naab, T. (2013). Naive Medientheorien und Third-Person Perception. Eine Untersuchung zur Integrierbarkeit beider Konzepte. Baden-Baden: Nomos.

Naab, T. K. (2012). The relevance of people's attitudes towards freedom of expression in a changing media environment. ESSACHESS - Journal for Communication Studies, 5(1), online only publication. http://www.essachess.com/index.php/jcs/article/view/150/136 [22.01.2016].

Naab, T. K. (im Druck). Soziale Kontrolle durch LaienkommunikatorInnen in sozialen Medien. In P. Werner, L. Rinsdorf, T. Pleil \& K.-D. Altmeppen (Hrsg.), Verantwortung - Gerechtigkeit Öffentlichkeit. Normative Perspektiven auf Kommunikation. Konstanz/München: UVK.

Neuwirth, K., \& Frederick, E. (2002). Extending the framework of third-, first-, and second-person effects. Mass Communication and Society, 5(2), 113-140.

Paek, H.-J., Lambe, J. L. \& McLeod, D. M. (2008). Antecedents to support for content restrictions. Journalism \& Mass Communication Quarterly, 85(2), 273-290.

Peffley, M., Knigge, P. \& Hurwitz, J. (2001). A multiple values model of political tolerance. Political Research Quarterly, 54(2), 379-406.

Price, V., Tewksbury, D. \& Huang, L.-N. (1998). Third-person effects on publication of a holocaust-denial advertisement. Journal of Communication, 48(2), 3-26.

Rojas, H., Shah, D. V. \& Faber, R. J. (1996). For the good of others: Censorship and the thirdperson effect. International Journal of Public Opinion Research, 8(2), 163-186.

Salwen, M. B. \& Dupagne, M. (1999). The third-person effect: Perceptions of the media's influence and immoral consequences. Communication Research, 26(5), 523-549.

Searing, D. D., Crewe, I. \& Conover, P. J. (2007). Political tolerance: Meaning, measurement and context. In J. Bara \& A. Weal (Eds.), Democratic politics and party competition (S. 287-311). New York, NY: Routledge.

Stevens, G., Agho, K., Taylor, M., Jones A. L., Jacobs, J., Barr, M. et al. (2011). Alert but less alarmed: a pool analysis of terrorism threat perception in Australia. BMC Public Health, 11, 797-808.

Stupi, E. K., Chiricos, T. \& Gertz, M. (2014). Perceived criminal threat from undocumented immigrants: Antecedents and consequences for policy preferences. Justice Quarterly, published online before print. http://www.tandfonline.com/doi/abs/10.1080/07418825.2014.902093\#. VckJ2Pntmko [22.01.2016].

Suedfeld, P., Steel, G. D. \& Schmidt, P. W. (1994). Political ideology and attitudes toward censorship. Journal of Applied Social Psychology, 24(9), 765-781.

Sullivan, J. L., Piereson, J. E. \& Marcus, G. E. (1982). Political tolerance and American democracy. Chicago, IL: University of Chicago Press.

Sullivan, J. L., Walsh, P., Shamir, M., Barnum, D. G. \& Gibson, J. L. (1993). Why politicians are more tolerant: Selective recruitment and socialization among political elites in Britain, Israel, New Zealand and the United States. British Journal of Political Science, 23(1), 51-76.

Sun, Y., Pan, Z. \& Shen, L. (2008). Understanding the third-person perception: Evidence from a meta-analysis. Journal of Communication, 58(2), 280-300.

Thompson, M. E. (1995). The impact of need for cognition on thinking about free speech issues. Journalism \& Mass Communication Quarterly, 72(4), 934-947.

Thompson, M. E., Chaffee, S. H. \& Oshagan, H. H. (1990). Regulating pornography: A public dilemma. Journal of Communication, 40(3), 73-83.

Trepte, S., Reinecke, L. \& Behr, K.-M. (2008). Qualitätserwartungen und ethischer Anspruch bei der Lektüre von Blogs und von Tageszeitungen. Publizistik, 53(4), 509-534.

Tsfati, Y. \& Cohen, J. (2003). On the effect of the "third-person effect": Perceived influence of media coverage and residential mobility intentions. Journal of Communication, 53(4), 711-727.

United Nations General Assembly (2011). United Nations General Assembly (2011). Report of the Special Rapporteur to the General Assembly on the right to freedom of opinion and expression exercised through the Internet (A/66/290). http://www.ohchr.org/EN/Issues/FreedomOpini on/Pages/Annual.aspx [24.01.2016]. 
van Eimeren, B. \& Frees, B. (2014). 79 Prozent der Deutschen online - Zuwachs bei mobiler Internetnutzung und Bewegtbild. Media Perspektiven, o. Jg.(7-8), 378-396.

Weck, A. (2012). Facebook und der Vorwurf der Zensur. http://www.netzpiloten.de/facebookund-der-vorwurf-der-zensur/ [22.01.2016].

Wei, R. \& Lo, V.-h. (2007). The third-person effects of political attack ads in the 2004 U.S. presidential election. Media Psychology, 9(2), 367-388.

Wei, R., Lo, V.-h. \& Lu, H.-Y. (2010). The third-person effect of tainted food product recall news: Examining the role of credibility, attention, and elaboration for college students in Taiwan. Journalism \& Mass Communication Quarterly, 87(3-4), 598-614.

Wu, W. \& Koo, S. H. (2001). Perceived effects of sexually explicit internet content: The third-person effect in Singapore. Journalism E Mass Communication Quarterly, 78(2), 260-274.

Youn, S., Faber, R. J. \& Shah, D. V. (2000). Restricting gambling advertising and the third-person effect. Psychology \& Marketing, 17(7), 633-649.

Zhao, X. \& Cai, X. (2008). From self-enhancement to supporting censorship: The third-person effect process in the case of internet pornography. Mass Communication and Society, 11(4), $437-$ 462.

Ziegele, M., Breiner, T. \& Quiring, O. (2014). What creates interactivity in online news discussions? An exploratory analysis of discussion factors in user comments on news items. Journal of Communication, 64(6), 1111-1138.

\section{Anhang}

Anhang 1: Items zur Messung des Sanktionsbedarfs

- Ich melde diese Gruppe, damit Facebook sie löscht.

- Ich melde diese Gruppe dem Gesetzgeber, damit er sie verbietet.

- Ich starte einen Aufruf auf Facebook, diese und andere Scientology-Gruppen zu melden.

- Facebook sollte die Gründerinnen und Gründer dieser Gruppe sperren.

- Facebook sollte die Gruppe löschen.

- Facebook sollte die Gruppe prüfen, bevor diese veröffentlicht werden darf.*

- Der Staat sollte die Gründerinnen und Gründer dieser Gruppe strafrechtlich verfolgen.

- Der Staat sollte Facebook zwingen, diese Gruppe zu löschen.

- Der Staat sollte die Gruppe prüfen, bevor diese veröffentlicht werden darf.*

*Aus der späteren Analyse ausgeschlossen 